\title{
Comparison of gel swelling under organic vapor and in organic solvent
}

\author{
M. Erdoğan ${ }^{1}$ and Ö. Pekcan ${ }^{2}$ \\ ${ }^{1}$ Department of Physics, Balikesir University, Balikesir, 10100, Turkey \\ 2 Department of Physics, Istanbul Technical University, Maslak, Istanbul, 80626, Turkey
}

\begin{abstract}
A Fast Transient Fluorescence Technique (FTRF) was employed for studying swelling of disc shaped poly (methyl methacrylate) (PMMA) gels, which were prepared by free radical copolymerization of methyl (methacrylate) (MMA) using various ethylene glycol dimethacrylate (EGDM) contents at $60{ }^{\circ} \mathrm{C}$. Pyrene (P) was introduced as a fluorescence probe during polymerization. Swelling experiments were performed by using P doped PMMA gels under chloroform vapor and in chloroform at room temperature. P lifetimes in and out of the gel were measured from fluorescence decay traces during in-situ swelling experiments. It was observed that P lifetimes in the gel decreased as swelling proceeds. An equation is derived for low quenching efficiencies to interpret the behavior of P lifetimes during swelling. The Li-Tanaka equation was used to determine the cooperative, $D_{c}$ diffusion coefficients for the gels made at various crosslinker contents. It is observed that $D_{c}$ values decrease as the crosslinker content is increased both in chloroform vapor and in chloroform.
\end{abstract}

\section{INTRODUCTION}

Gels are known to exist generally in two forms, swollen or shrunken. Volume phase transitions occur between these forms either continuously or as sudden jumps between them [1, 2]. The equilibrium swelling of gels in solvents has been extensively studied [3, 4]. The swelling kinetics of physical and chemical gels are very important in many technological applications. Especially in pharmaceutical industries for designing slowrelease devices for oral drugs. In agricultural industry for producing storable foods and in medical applications for developing artificial organs the knowledge of the volume transitions of gels are quite important.

Swelling is directly related to the viscoelastic properties of a gel. The gel elasticity and the friction between the network and solvent play an important role in the kinetics of gel swelling [5-7]. It is known that the relaxation time of swelling is proportional to the square of a linear size of the gel [5]. A fact that has been confirmed experimentally [7]. One of the most important features of the gel swelling process is that it is isotropic, i.e., when the radius increases $10 \%$, the axial length increases $10 \%$ in a long cylindrical gel. The elastic and swelling properties of permanent networks can be understood by considering two opposing effects, the osmotic pressure and the restraining force. Usually the total free energy of a chemically crosslinked network can be separated into two terms; the bulk and the shear energies. The bulk energy of the system is related to the volume change, which is controlled by diffusion. The other important energy, the shear energy, keeps the gel in shape by minimizing the nonisotropic deformation [8, 9]. Li and Tanaka [10] have developed a model where the shear modulus, $\mu$ plays an important role that keeps the gel in shape due to coupling of any changes in different directions. This model predicts that the geometry of the gel is an important factor, and swelling is not just a diffusion process.

Several experimental techniques have been employed to study the kinetics of swelling, shrinking and drying of chemical and physical gels, among which are neutron scattering [11], quasielastic light-scattering [12], macroscopic experiments [13] and in-situ interferometric measurements. Using a fluorescence technique, a P derivative was employed as a fluorescence probe to monitor the polymerization, aging and drying of aluminosilicate gels [14], with peak ratios in emission spectra being monitored during these processes. Steady-state fluorescence (SSF) measurements on the swelling of gels formed by the FCC of methyl methacrylate (MMA) and ethylene glycol dimethacrylate (EGDM) in solution have been reported. A P derivative was used as a fluorescence probe to monitor swelling, desorption and drying in real time during in-situ fluorescence experiments $[15,16]$. Time-resolved and steady-state fluorescence techniques were employed to study isotactic polystyrene in its gel state [17] where excimer spectra were used to monitor the existence of two different conformations in the gel state of polystyrene. Recently, fast a transient fluorescence (FTRF) technique was used for monitoring swelling of PMMA gels in solution [18].

The purpose of this work is to study gel swelling at the molecular level, where excited P molecules are quenched by the penetrating organic molecules in the range of a few Ångstroms. The penetration of organic molecules into disc shaped gels formed by FCC of MMA and various amounts of EGDM was studied using a Fast Transient Fluorescence (FTRF) technique which measures lifetimes. Fluorescence decay profiles of $\mathrm{P}$ were 
measured when the gel was illuminated directly by the exciting light and decay profiles were fitted to an exponential law to obtain lifetimes of P. Measuring lifetimes directly provides swelling parameters. It was observed that as the gel swells, the lifetime of $\mathrm{P}$ inside the gel decreases which can be modeled using a low quenching Stern-Volmer equation. Cooperative, $D_{c}$ diffusion coefficients were determined for the gels at various crosslinker contents by employing Li-Tanaka and Stern-Volmer equations and found to decrease from 4 to $1.8 \times 10^{-5} \mathrm{~cm}^{2} \mathrm{~s}^{-1}$ under chloroform vapor and from 2.5 to $1.68 \times 10^{-5} \mathrm{~cm}^{2} \mathrm{~s}^{-1}$ in chloroform by increasing the EGDM content from 0.015 to 0.035 vol.\%.

\section{KINETICS OF SWELLING}

Swelling experiments of disc shaped gels have shown that the relative changes of diameter and thickness are the same, indicating that the gel-swelling processes are not pure diffusional processes. In fact the equality of the relative changes of diameter and thickness stems from the non zero shear modulus, $\mu$ which results; the change of the total shear energy in response to any small change in shape that maintains constant volume element within the gel should be zero. The high friction coefficient, $f$, between the network and the solvent overdamps the motion of the network, resulting in a diffusion-like relaxation. The equation of the motion of a network element during the swelling can be given by [10]

$$
\frac{\partial \vec{u}}{\partial t}=D_{c} \vec{\nabla}^{2} \vec{u}
$$

where $\vec{u}$ is the displacement vector measured from the final equilibrium location after the gel is fully swollen $(u=0$ at $t=\infty) \cdot D_{c}=(K+4 \mu / 3) / f$ is the collective diffusion coefficient. Here $t$ denotes the time and $K$ is the bulk modulus. Equation (1) has been used with some success to study the swelling of gels [5]. However, these studies did not properly treat the shear deformation that occurs within a gel during swelling, and, hence, cannot explain, for example, the isotropic swelling of a cylindrical gel. This shortcoming was due to the shear modulus of the network keeping the system in shape by minimizing the non-isotropic deformation. For a disc shaped gel, any change in diameter is coupled to a change in thickness. The total energy of a gel can be separated into a bulk energy and a shear energy. The bulk energy is related to the volume change, which is controlled by diffusion. The shear energy, $F_{s h}$ on the other hand, can be minimized instantly by readjusting the shape of the gel [10].

$$
\delta F_{s h}=0
$$

Each small diffusion process determined by eq. (1) must couple to a small shear process given by eq. (2) producing the following relation for a disc shaped gel

$$
\frac{u_{r}(r, t)}{r}=\frac{u_{z}(a, t)}{a}
$$

where $r$ is the radius and $a$ is the half thickness of the disc gel. Equation (3) indicates that the relative change in shape of the gel is isotropic, i.e., the swelling rates of a disc in the axial $(z)$ and radial $(r)$ directions are the same.

Simultaneous solution of Eqs. (1) and (2) produces the following equations for the swelling of a gel disc in axial and radial directions [10].

$$
\begin{aligned}
& u_{z}(z, t)=u_{z}(z, \infty) \sum_{n} B_{n} \exp \left(-t / \tau_{n}\right) \\
& u_{r}(r, t)=u_{r}(r, \infty) \sum_{n} B_{n} \exp \left(-t / \tau_{n}\right)
\end{aligned}
$$

where the axial and the radial displacements are expressed as series of components, each of them decaying exponentially with a time constant, $\tau_{n}$. The first terms of the expressions are dominant at large $t$, that is at the last stage of swelling. Equation (4) can also be written in terms of vapor and solvent uptakes $W$ and $W_{\infty}$ at time $t$ and at equilibrium, respectively, as follows

$$
\frac{W_{\infty}-W}{W_{\infty}}=\sum_{n=1}^{\infty} B_{n} \exp \left(-t / \tau_{n}\right)
$$

In the limit of large $t$, or if $\tau_{c}$ is much larger than the rest of $\tau_{n}$, all higher terms ( $\left.n \geq 2\right)$ in eq. (5) can be omitted and the swelling kinetics is given by the following relation

$$
\left(1-\frac{W}{W_{\infty}}\right)=B_{1} \exp \left(-t_{s} / \tau_{c}\right)
$$

It should be noted from eq. (5) that $\sum B_{n}=1$, therefore $B_{1}$ should be less than $1 . B_{1}$ is related to the ratio of the shear modulus, $\mu$, and longitudinal osmotic modulus, $M=(K+4 \mu / 3)$. Hence, once the value of $B_{1}$ is obtained, one can determine the value of $R=\mu / M$. Here we have to note that eq. (6) can also be obtained by using the theoretical results [10], in the case of $R \rightarrow 3 / 4(\mu / K \rightarrow$ $\infty)$, time constant $\tau_{c} \approx(3 / 4-R)^{-1}$ goes to infinity and all $B_{n}$ 's go to zero except $B_{1}$, which goes to unity. The dependence of $B_{1}$ on $R$ for a disc can be found in the literature [10]. $\tau_{c}$ is related to the collective diffusion coefficient $D_{c}$ at the surface of a gel disc by

$$
D_{c}=\frac{3 a^{2}}{T_{c} \alpha_{1}^{2}}
$$

where $\alpha_{1}$ is a function of $R$ only and is given in the literature [10], and $a$ stands for the half thickness of the gel in the final equilibrium state. Hence, $D_{c}$ can be calculated. 


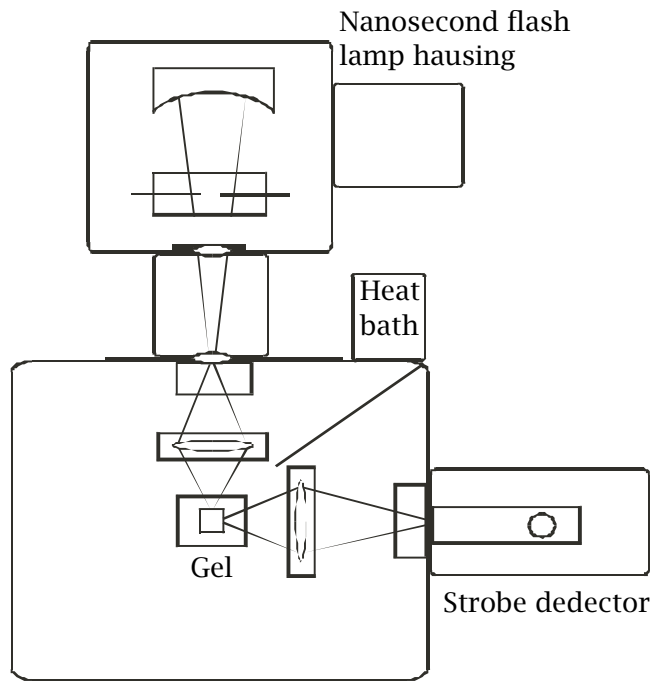

Sample compartment

Figure 1. Strobe master system of Photon Technology International (PTI) for fluorescence lifetime measurements.

\section{EXPERIMENTS}

The radical copolymerization of MMA and EGDM was performed in bulk at $60^{\circ} \mathrm{C}$ in the presence of $2,2^{\prime}$ azobisisobutyrronitrile (AIBN) as an initiator. $\mathrm{P}$ was added as a fluorescence probe during the gelation process. AIBN (0.26 wt\%) was dissolved in MMA and this stock solution was divided and transferred into round glass tubes of $9.5 \mathrm{~mm}$ internal diameter. All samples were deoxygenated by bubbling nitrogen for $10 \mathrm{~min}$ utes, and then radical copolymerization of MMA with five different amount of EGDM was performed to produce five different gels. Here, the $\mathrm{P}$ concentration was taken as $4 \times 10^{-4} \mathrm{M}$. Before use, the monomers MMA (Merck) and EGDM (Merck) were freed from the inhibitor by shaking with a $10 \%$ aqueous $\mathrm{KOH}$ solution, washing with water, and drying over sodium sulfate. They were then distilled under reduced pressure over copper chloride.

Fluorescence decay experiments were performed using a Photon Technology International (PTI) Strobe Master System (SMS) shown in Figure 1. In the strobe, or pulse sampling technique $[19,20]$ the sample is excited with a pulsed light source. The name comes about because the Photo Multiplier Tube (PMT) is gated or strobed by a voltage pulse that is synchronized with the pulsed light source. The intensity of fluorescence emission is measured in a very narrow time window after each pulse and saved in a computer. The time window is shifted after each pulse. The strobe has the effect of turning of the PMT and measuring the emission intensity over a very short time window. When the data have been sampled over the appropriate range of time, a decay curve of fluorescence intensity versus time can
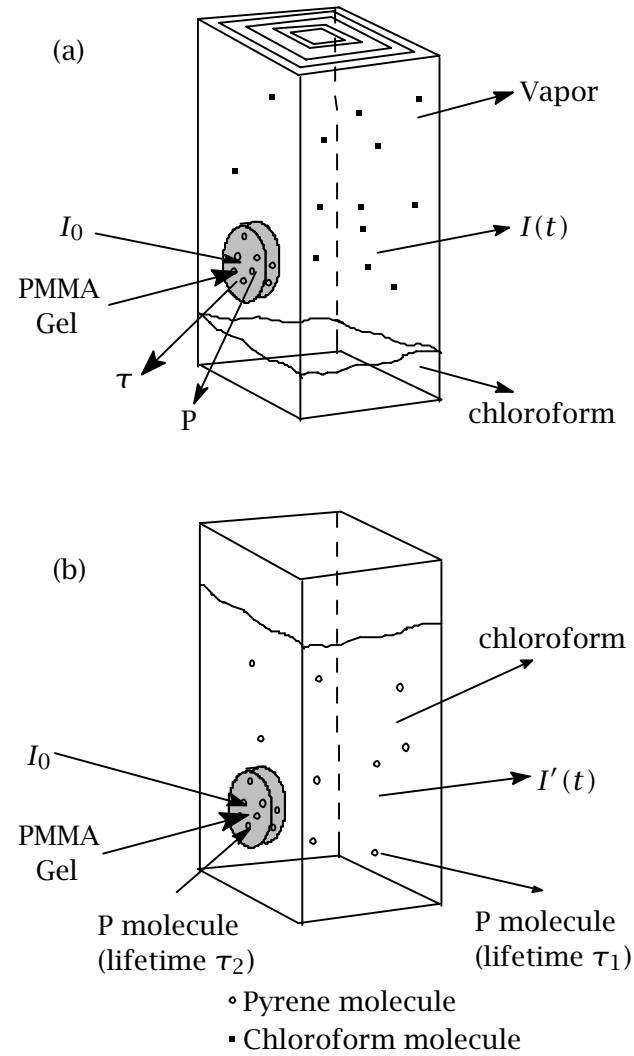

Figure 2. Fluorescence cell in PTI strobe master system during (a) vapor, (b) solvent induced swelling. $I_{0}, I(t)$, and $I^{\prime}(t)$ are the excitation and the emission intensities at 345 and $395 \mathrm{~nm}$, respectively. $\tau$ is the P lifetime for vapor induced gel swelling and $\tau_{2}$ and $\tau_{1}$ are lifetimes of $P$ in and out of the gel sample in solvent.

be constructed. Since the strobe technique is intensitydependent, a strobe instrument is much faster than SPC, and even faster than a phase instrument. A strobe instrument is much simpler to use than SPC and the data are easier to interpret than those from a phase system. Because of these advantages SMS is used here to monitor swelling of PMMA gels which takes around several hours.

In-situ swelling experiments were carried out in the SMS employing a pulsed lamp source $\left(0.5 \mathrm{~atm}\right.$ of $\mathrm{N}_{2}$ ). Pyrenes were excited at $345 \mathrm{~nm}$ and fluorescence decay curves were obtained at $395 \mathrm{~nm}$ during in-situ swelling experiments which were performed for various gels at different EGDM content at room temperature. The disc shaped gel sample was placed in a $1 \mathrm{~cm} \times 1 \mathrm{~cm}$ quartz cell, where it was attached to one side of the cell by pressing the disc with a thick steel wire. For the swelling experiments, at first the lower part of the quartz cell was filled with chloroform for vapor induced swelling (see Figure 2a) and second, the quartz cell was filled with chloroform itself as shown in Figure $2 b$. The cell 
Table 1.

\begin{tabular}{lcccccc}
\hline EGDM (vol.\%) & $\Delta \mathrm{m}(\mathrm{g})$ & $\Delta \mathrm{d}(\mathrm{cm})$ & $\tau_{c}(\mathrm{~s})$ & $B_{1}$ & $\alpha_{1}$ & $D_{c}\left(\mathrm{~cm}^{2} \mathrm{~s}^{-1}\right) \times 10^{-5}$ \\
\hline 0.015 & 0.20 & 0.10 & 3509 & 0.92 & 1,1 & 2.16 \\
0.020 & 0.20 & 0.09 & 6451 & 0.84 & 1.0 & 1.62 \\
0.025 & 0.24 & 0.10 & 7499 & 0.93 & 1.0 & 1.33 \\
0.030 & 0.15 & 0.08 & 7560 & 0.93 & 1.0 & 1.18 \\
0.035 & 0.19 & 0.11 & 6900 & 0.87 & 1,3 & 1.03 \\
\hline
\end{tabular}

$\Delta \mathrm{m}$; amount of vapor uptake,

$\Delta \mathrm{d}$; variation in disc thicknesses,

$\tau_{c}$; time constant

$D_{c}$; cooperative diffusion coefficient,

$B_{1}$ and $\alpha_{1}$; experimentally determined coefficients,

Experimentally obtained parameters for the gel swelling under chloroform vapor.

Table 2 .

\begin{tabular}{lcccccc}
\hline EGDM (vol.\%) & $\Delta \mathrm{m}(\mathrm{g})$ & $\Delta \mathrm{d}(\mathrm{cm})$ & $\tau_{c}(\mathrm{~s})$ & $B_{1}$ & $\alpha_{1}$ & $D_{c}\left(\mathrm{~cm}^{2} \mathrm{~s}^{-1}\right) \times 10^{-5}$ \\
\hline 0.015 & 0.27 & 0.13 & 1980 & 0.80 & 1.6 & 2.49 \\
0.020 & 0.40 & 0.12 & 2955 & 0.85 & 1.4 & 2.07 \\
0.025 & 0.23 & 0.12 & 5040 & 0.91 & 1.1 & 1.78 \\
0.030 & 0.28 & 0.11 & 4740 & 0.92 & 1.1 & 1.42 \\
0.035 & 0.16 & 0.10 & 5310 & 0.92 & 1.1 & 1.60 \\
\hline
\end{tabular}

$\Delta \mathrm{m}$; amount of vapor uptake,

$\Delta \mathrm{d}$; variation in disc thicknesses,

$\tau_{c}$; time constant,

$D_{c}$; cooperative diffusion coefficient,

$B_{1}$ and $\alpha_{1}$; experimentally determined coefficients,

Experimentally obtained parameters for the gel swelling in chloroform.

was placed in the SMS system where fluorescence decay measurements were performed at $90^{\circ}$ angle. In swelling experiments five identical disc shaped gels were used which were dried and cut from a cylindrical gel obtained from FCC with various EGDM content. The fluorescence decay data were collected over 3 decades of decay and fitted by nonlinear least squares using a deconvolution method with a dry gel as a scatterer standard. The uniqueness of the fit of the data to the model is determined by $\chi^{2}\left(\chi^{2} \leq 1.10\right)$, the distribution of the weighted residuals and the autocorrelation of the residuals. Macroscopic vapor and solvent uptake, $\Delta \mathrm{m}$ and disc thickness, $\Delta \mathrm{d}$ measurements were performed using of microbalance and calipers, respectively, and results are listed in Tables 1 and 2, respectively.

\section{RESULTS AND DISCUSSIONS}

Decay curves of P obtained from SMS at various swelling times, $t_{s}$ for the gel sample prepared with 0.015 EGDMvol.\% are presented in Figures $3 a$ and $b$ for vapor and solvent induced swellings, respectively. In order to probe the swelling process during vapor uptake, the fluorescence decay curves are measured when the gel is in the position of Figure 2a and they were fitted to the following exponential law:

$$
I(t)=A e^{-t / \tau}
$$

where $\tau$ is the pyrene lifetime and $A$ is the corresponding amplitude of the decay curve. As seen in Figure 3a pyrene decay faster as the swelling time, $t_{s}$ is increased, indicating that pyrenes are quenched by the chloroform molecules. Similarly, to probe the swelling process during solvent uptake, the fluorescence decay curves were measured when the gel was in the position of Figure $2 b$, and they were fitted to the sum of two exponentials:

$$
I^{\prime}(t)=A_{1} e^{-t / \tau_{1}}+A_{2} e^{-t / \tau_{2}}
$$

where $\tau_{1}$ and $\tau_{2}$ are the long and short components of pyrene lifetimes and $A_{1}$ and $A_{2}$ are the corresponding amplitudes of the decay curves. In other words, $\tau_{2}$ and $\tau_{1}$ are the lifetimes of $\mathrm{P}$ when the pyrenes are in and out of the gel sample, respectively. Figure $3 \mathrm{~b}$ demonstrates that as the swelling time, $t_{s}$ is increased excited pyrenes decay faster and faster which indicates that as solvent uptake is increased quenching of excited pyrenes increases. $\tau$ values for vapor induced gel swelling and $\tau_{1}$ and $\tau_{2}$ values for solvent induced gel swelling are plotted versus $t_{s}$ in Figures $4 \mathrm{a}$ and $\mathrm{b}$, respectively, for the gels prepared with 0.015 EGDMvol.\% content. It is seen in Figure 4 that, $\tau_{1}$ values do not change much however $\tau$ and $\tau_{2}$ values decrease as $t_{s}$ is increased. Here the role of the vapor and solvent is to add the quasicontinuum of states needed to satisfy energy resonance conditions, i.e., the vapor and solvent act as an energy sink for rapid vibrational relaxation, which occurs after the rate limiting transition from the initial state. Birks et al. studied the influence of solvent viscosity on the fluorescence characteristics of pyrene solutions in 

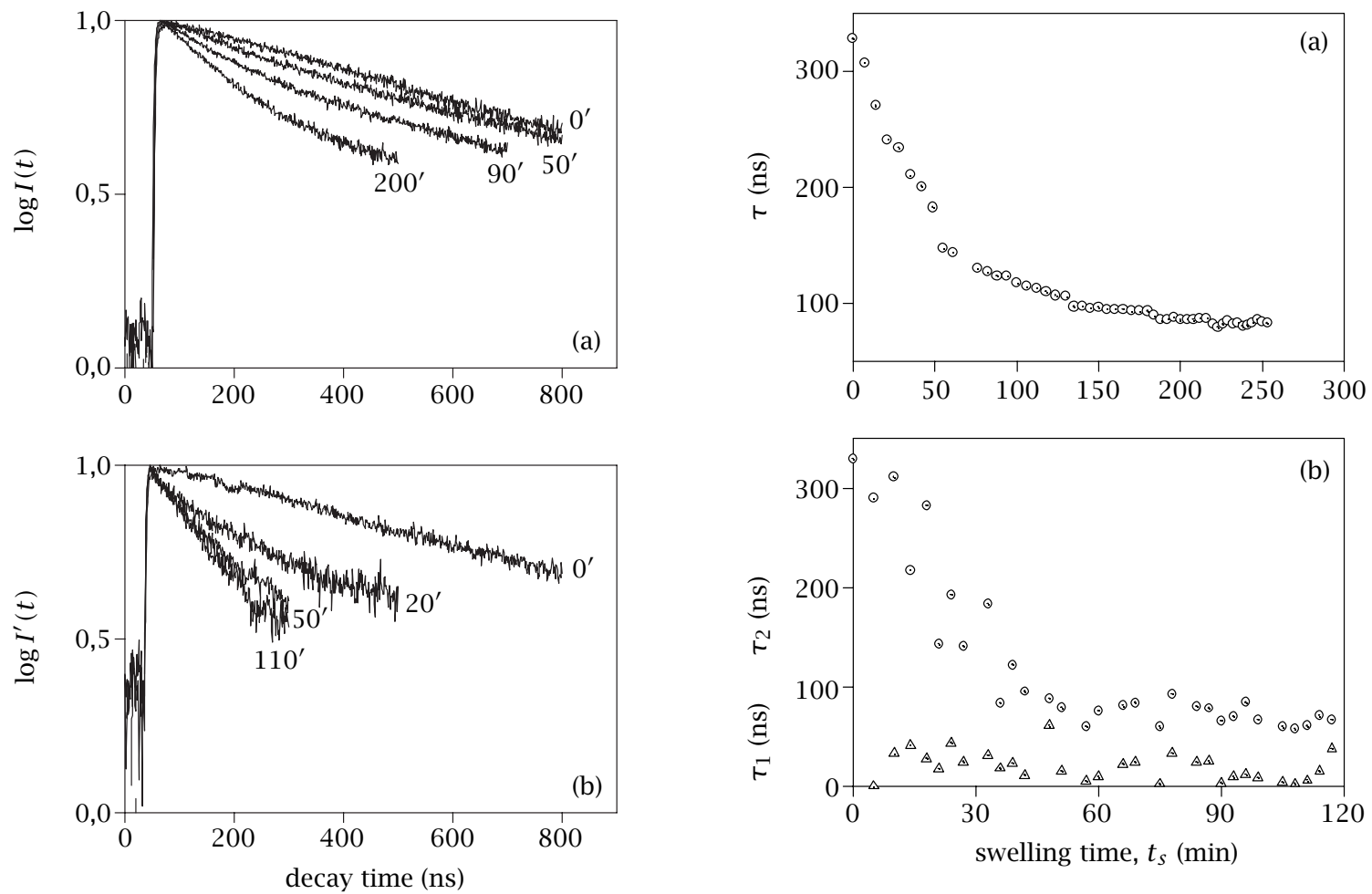

Figure 3. Log of the fluorescence decay profiles, $I(t)$ and $I^{\prime}(t)$ of $P$ at various swelling times for the gel sample prepared with 0.015 vol.\% EGDM content for (a) vapor, (b) solvent, induced gel swelling. The number on each decay curve presents the swelling times, $t_{s}$ in minute.

various solvents and observed that the rate of monomer internal quenching is affected by solvent quality [21].

In order to quantify the results in Figure 4 where exponential decrease in $\tau$ and $\tau_{2}$ are observed as the swelling time, $t_{s}$ is increased, the Stern-Volmer type of quenching mechanism may be proposed for the fluorescence decay of $\mathrm{P}$ in the gel sample. According to the Stern-Volmer law, in general $\tau$ lifetimes can be written as [21]:

$$
\tau^{-1}=\tau_{0}^{-1}+k[W]
$$

where $\tau_{0}$ is the lifetime of $\mathrm{P}$ in the dry gel in which no quenching has taken place, $k$ is the quenching rate constant and $[W]$ is the vapor or solvent concentration in the gel after vapor or solvent uptake has started. For low quenching efficiency, where $\tau_{0} k[W]<1$, eq. (10) becomes

$$
\tau \approx \tau_{0}\left(1-\tau_{0} k[W]\right)
$$

If one integrates eq. (11) over the differential volume $d v$ of the gel from its initial, to final thickness, the following relation is obtained.

$$
W=\left(1-\frac{\tau}{\tau_{0}}\right) \frac{v}{k \tau_{0}}
$$

Figure 4. The plots of the measured lifetime values versus swelling time, $t_{s}$ for $0.015 \mathrm{vol} . \%$ EGDM content gel, (a) $\tau$ for vapor, (b) $\tau_{1}$ and $\tau_{2}$ for solvent induced gel swelling.
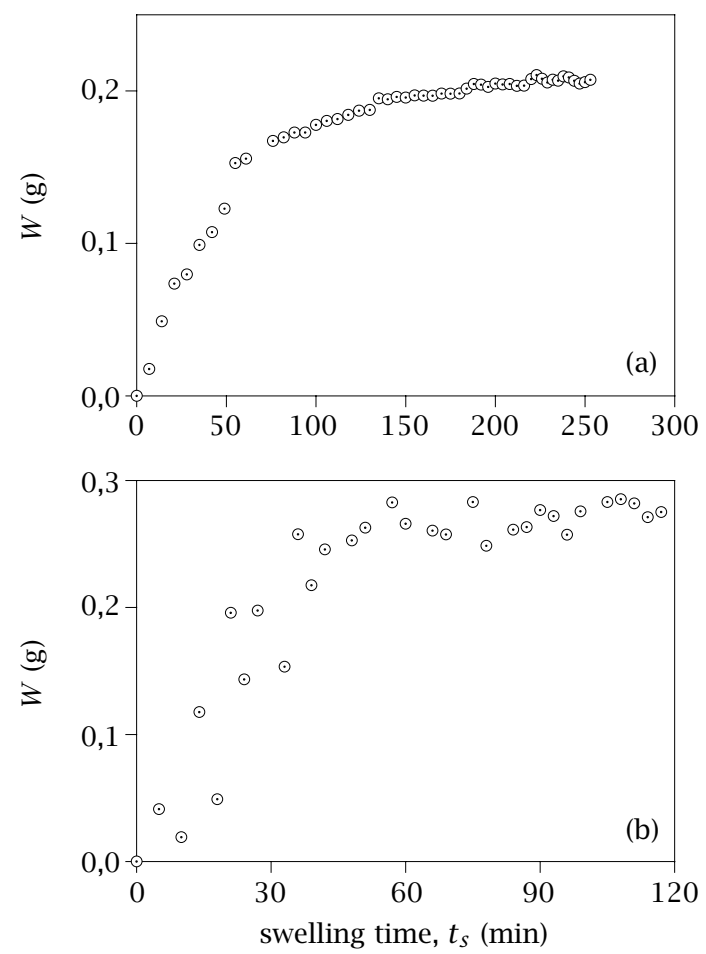

Figure 5. The plots of (a) vapor, (b) solvent, uptake $W$ versus swelling time, $t_{s}$ for $0.015 \mathrm{vol} . \%$ EGDM content gel sample. 

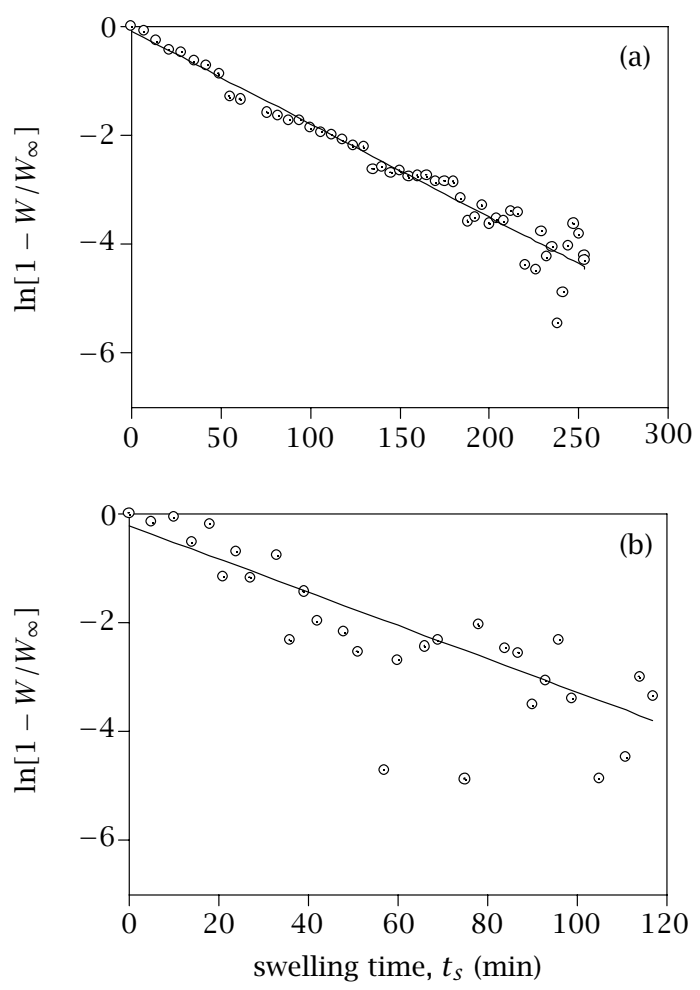

Figure 6. Fit of the data in Figure 5 to eq. (6), where the slope of the curves produced $\tau_{c}$ values which are listed in Tables 1 and 2 for vapor and solvent induced gel swelling, respectively.

Here vapor or solvent uptake, $W$ is calculated over differential volume, $d v$ as

$$
W=\int_{i}^{f}[W] d v
$$

Where $v$ is the swollen volume of the gel, which can be measured experimentally. $k$ was obtained from separate measurements by using eq. (12), where the infinity equilibrium value of vapor or solvent uptake, $W_{\infty}$ was used for calculation. Since $\tau_{0}(\approx 300 \mathrm{~ns})$ is already known from the dry gel, and measured values of $v$ and $\tau$ at equilibrium swelling condition can be used to calculate $k$ for each swelling experiments separately. The plots of the vapor and solvent uptake, $W$ for the gels prepared with 0.015 vol.\% are shown in Figures 5a and b, respectively, which are typical vapor and solvent uptake curves, obey the Li-Tanaka equation (eq. (6)).

In a logarithmic form the data of Figure 5 can be fitted to the following relation derived from eq. (6).

$$
\ln \left(1-\frac{W}{W_{\infty}}\right)=\ln B_{1}-\frac{t_{s}}{\tau_{c}}
$$

The fits are presented in Figures $6 \mathrm{a}$ and $\mathrm{b}$ for vapor and solvent induced gel swelling, respectively. From these $B_{1}$ and $\tau_{c}$ values have been calculated as listed in Tables 1 and 2, respectively. The plot of $\tau_{\mathcal{C}}$

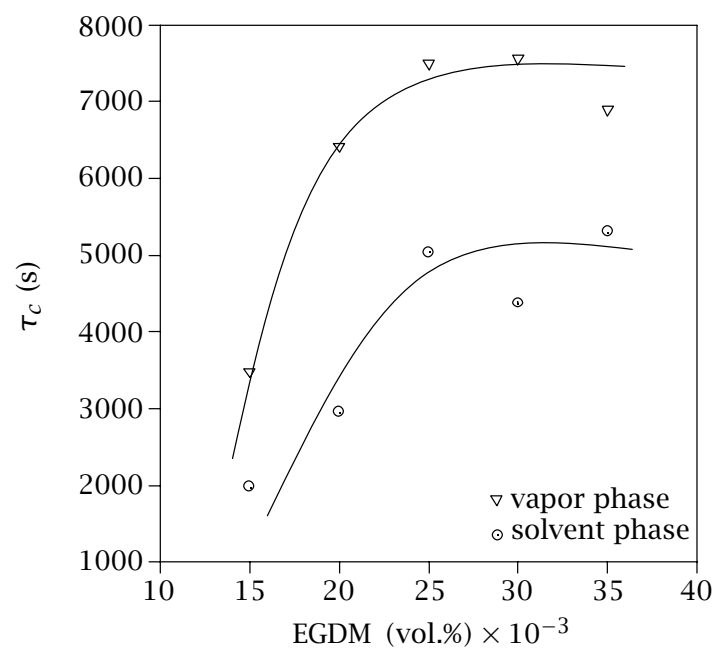

Figure 7. The plots of $\tau_{c}$ values versus vol.\% EGDM content for vapor and solvent induced gel swelling. Symbols, $\nabla$ and $\odot$ stand for vapor and solvent induced gel swelling, respectively.

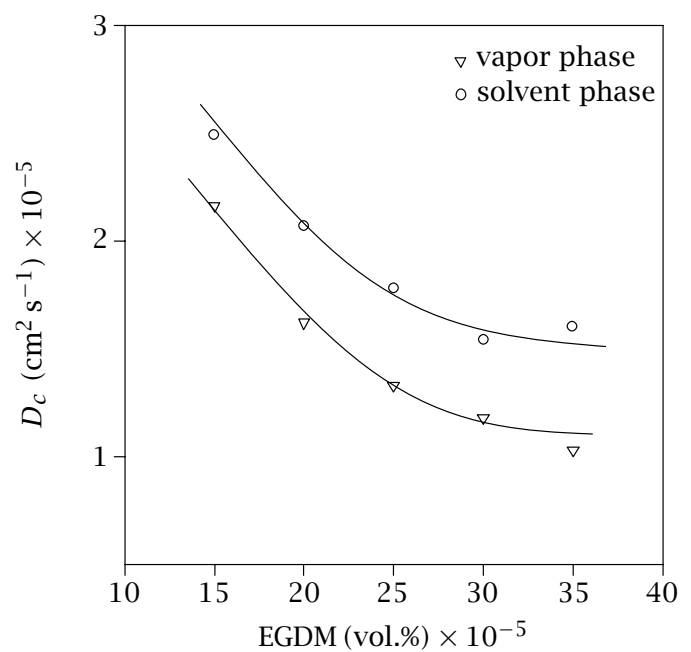

Figure 8. The plots of $D_{c}$ values versus vol.\% EGDM content for vapor and solvent induced gel swelling. Symbols, $\nabla$ and o stand for vapor and solvent induced gel swelling, respectively.

versus EGDM content is shown in Figure 7 for vapor and solvent induced swelling, where it is seen that as the EGDM content is increased penetration of vapor and solvent molecules slows down, as expected. In other words chloroform molecules penetrate into the densely formed gels much slower than into loosely formed gels in both cases. Penetration of chloroform vapor is much slower than that of liquid chloroform most probably due to a lower osmotic pressure in the vapor phase.

By knowing $B_{1}$ values one can obtain $\alpha_{1}$ values and then from eq. (7) $D_{c}$ values are obtained and are plotted in Figure 8 versus EGDM content for vapor and 
solvent induced swelling. The behaviour of $D_{c}$ in Figure 8 predicts that as the EGDM content increases $D_{c}$ values decrease, i.e., gel segments move much faster in loosely formed gels than in densely formed gels.

\section{CONCLUSION}

In this paper we have shown that the FTRF technique can be used to measure time constants, $\tau_{c}$ and cooperative diffusion coefficients, $D_{c}$ as a function of the EGDM content during polymeric gel swelling induced by organic vapor and organic solvent. It is understood that organic molecules penetrate much slower from the vapor phase than from a liquid solvent into PMMA gels. It is also shown that penetration of organic molecules is much faster in loosely formed gels than in densely formed gels from both phases. It is seen in Tables 1 and 2 that macroscopic measurements such as $\Delta \mathrm{m}$ and $\Delta \mathrm{d}$ are not very sensitive to the environment of the gel, i.e., similar $\Delta \mathrm{m}$ and $\Delta \mathrm{d}$ values were produced in both vapor and solvent phases. $\Delta \mathrm{m}$ and $\Delta \mathrm{d}$ values are also independent of the EGDM content in PMMA gels. Here it can be concluded that the FTRF technique can be used to study gel swelling under various internal and external conditions with a quite high accuracy.

\section{REFERENCES}

[1] K. Dusek and D. Peterson, J. Poly. Sci. A2 (1968), 1209.

[2] T. Tanaka, Phys. Rev. Lett. 45 (1980), 1636.

[3] A. V. Tobolsky and J. C. Goobel, Macromolecules 3 (1970), 556.

[4] H. G. Schild, Prog. Polym. Sci. 17 (1992), 163.

[5] T. Tanaka and D. Filmore, J. Chem. Phys. 70 (1979), 1214.
[6] A. Peters and S. J. Candau, Macromolecules 19 (1986), 1952.

[7] P. Chiarelli and D. De Rossi, Progr. Colloid Polym. Sci. 78 (1988), 4.

[8] K. Dusek and W. Prins, Adv. Polym. Sci. 6 (1969), 1.

[9] S. Candau, J. Bastide, and M. Delsanti, Adv. Polym. Sci. 7 (1982), 44.

[10] Y. Li and T. Tanaka, J. Chem. Phys. 92(2) (1990), 1365.

[11] J. Bastide, R. Duoplessix, C. Picot, and S. J. Candau, Macromolecules 17 (1984), 83.

[12] A. Peters and S. J. Candau, Macromolecules 21 (1988), 2278.

[13] C. Wu and C. Y. Yang, Macromolecules 27 (1994), 4516.

[14] J. C. Panxviel, B. Dunn, and J. J. Zink, J. Phys. Chem. 93 (1989), 2134.

[15] Y. Yilmaz and Ö. Pekcan, Polymer 39 (1998), 5351.

[16] Ö. Pekcan and Y. Yilmaz, Applied Fluorescence in Chemistry, Biology and Medicine (W. Rettig and B. Strehnnel, eds.), Springer-Verlag, Berlin, 1999, p. 331.

[17] B. Wandelt, D. J. S. Birch, R. E. Imhof, A. S. Holmes, and R. A. Pethnick, Macromolecules 24 (1991), 5141.

[18] Ö. Pekcan, D. Kaya, and M. Erdoğan, Polymer 41 (2000), 4915.

[19] J. R. Lakowicz, Principles of Fluorescence Spectroscopy, Plenum Press, New York, 1983.

[20] W. R. Ware, D. R. James, and A. Siemianczuk, Rev. Sci. Inst. 63 (1992), 1710.

[21] J. B. Birks, M. D. Lumb, and J. H. Mumra, Proc. R. Soc. Sev. A. 277 (1989), 289. 


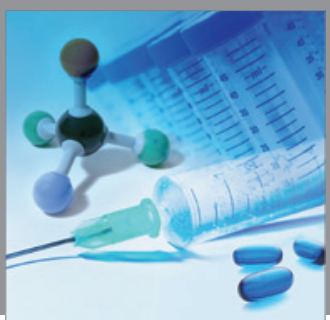

International Journal of

Medicinal Chemistry

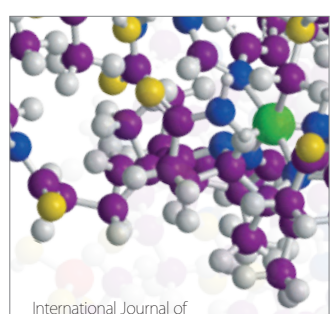

Carbohydrate Chemistry

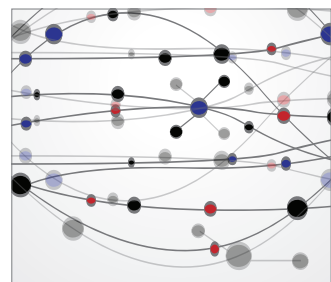

The Scientific World Journal
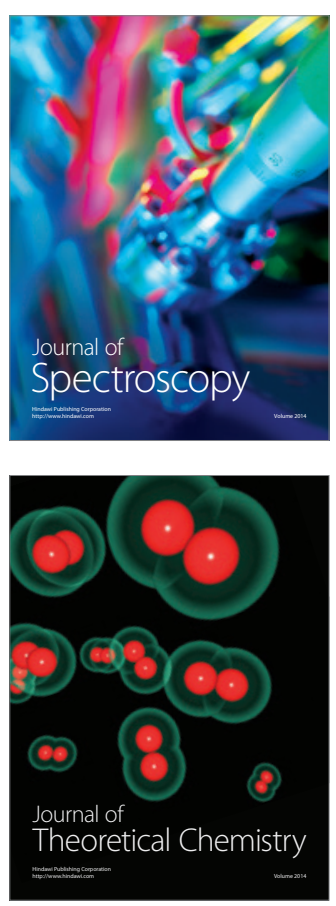
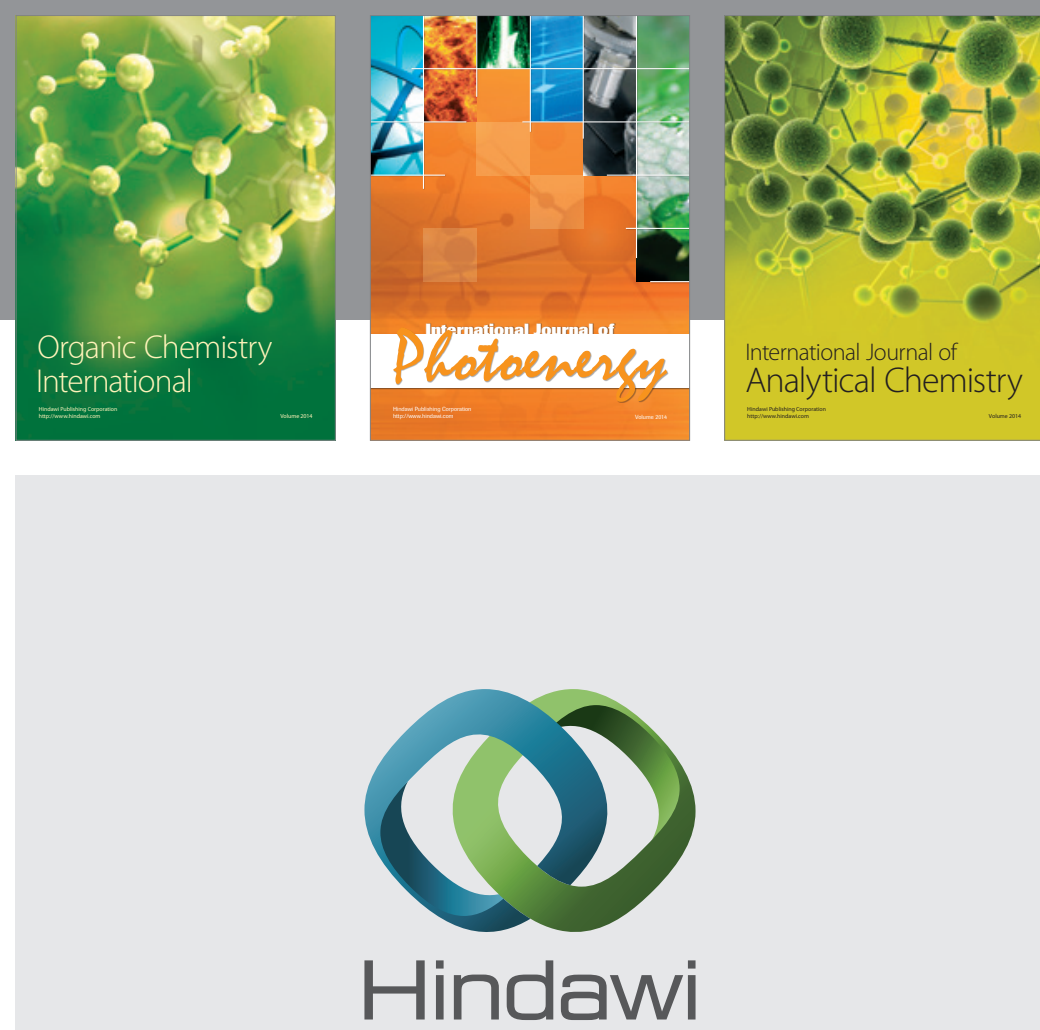

Submit your manuscripts at

http://www.hindawi.com
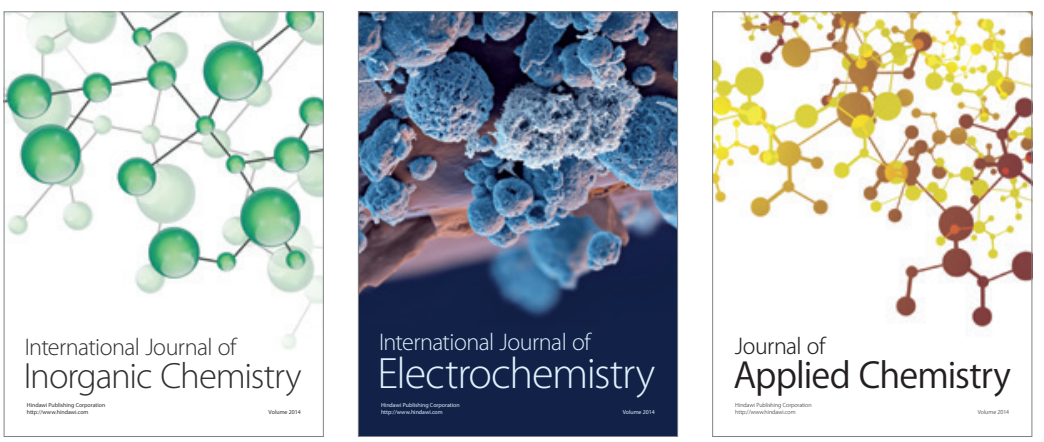

Journal of

Applied Chemistry
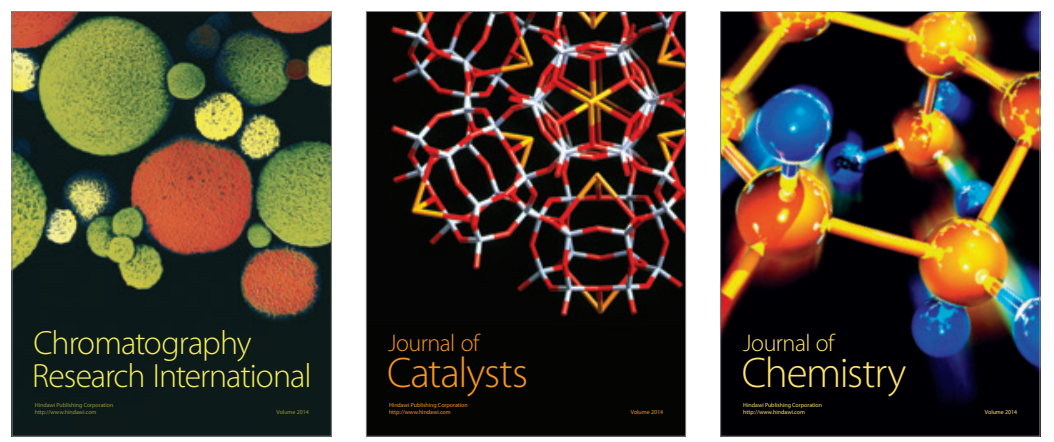
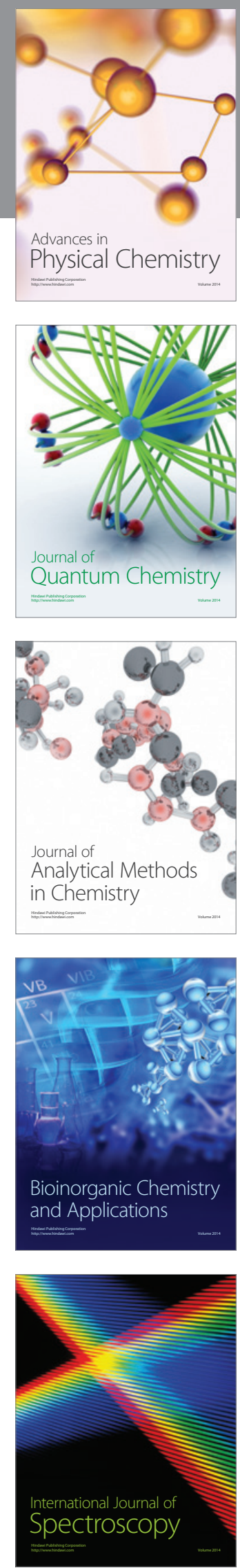